\title{
Efeito da carga de gemas da videira 'Cabernet Franc' na interceptação da radiação solar e na fertilidade de gemas
}

\author{
Effect of retained node numbers of Cabernet Franc grapevine on the interception of solar \\ radiation and on the node fertility
}

\section{Douglas André Würz ${ }^{1 *}$, Bruno Farias Bonin ${ }^{2}$, Alberto Fontanella Brighenti ${ }^{3}$, Adrielen Tamiris Canossa' ${ }^{2}$, Juliana Reinehr ${ }^{2}$, Ricardo Allebrandt ${ }^{2}$, Betina Pereira de Bem ${ }^{2}$, Leo Rufato ${ }^{2}$ e Aike Anneliese Kretzschmar ${ }^{2}$}

${ }^{1}$ Instituto Federal de Santa Catarina, Canoinhas, SC, Brasil. *Autor para correspondência: douglaswurz@hotmail.com.

${ }^{2}$ Universidade do Estado de Santa Catarina, Lages, SC, Brasil.

${ }^{3}$ Empresa de Pesquisa Agropecuária e Extensão Rural de Santa Catarina, São Joaquim, SC, Brasil.

Submissão:16/11/2018 / Aceite: 01/07/2019

\begin{abstract}
RESUMO
Devido a uma carência de informações técnico-cientificas sobre o efeito da carga de gemas em relação a interceptação da radiação solar, e o seu efeito na fertilidade de gemas, tem-se como objetivo desse trabalho avaliar o efeito do aumento da carga de gemas da videira 'Cabernet Franc' em relação a interceptação da radiação fotossinteticamente ativa e o seu efeito na fertilidade de gemas em região de elevada altitude de Santa Catarina. Este experimento foi conduzido durante safra 2016/2017, em um vinhedo comercial, localizado no munícipio de São Joaquim. Utilizaram-se plantas de 'Cabernet Franc' enxertadas sobre o porta-enxerto 'Paulsen 1103'. Os tratamentos consistiram em quatro diferentes níveis de cargas de gemas: 15, 30, 50 e 75 gemas planta $^{-1}$. O delineamento experimental utilizado foi o de blocos ao acaso, com quatro blocos e cinco plantas por parcela. Os dados foram submetidos à análise de variância (ANOVA) e comparados pelo Teste Tukey a 5\% de probabilidade de erro. Os resultados desse estudo evidenciam a importância da interceptação da radiação fotossinteticamente ativa na fertilidade de gemas, observando-se redução da fertilidade de gemas em situações de redução da interceptação da radiação fotossinteticamente ativa, nesse caso, causado pelo aumento da carga de gemas, que resulta em um maior número de ramos, proporcionando um dossel mais denso, reduzindo a interceptação da radiação fotossinteticamente ativa que alcança as gemas da videira, sendo esse efeito, mais pronunciado nas gemas localizada na posição basal do ramo.
\end{abstract}

PALAVRAS-CHAVE: Vitis vinifera L., poda, equilíbrio vegetativo, manejo do dossel.

\section{ABSTRACT}

Due to a lack of technical-scientific information about the effect of the retained node number on the interception of the solar radiation of the nodes and its effect on the fertility of nodes, this work aims to evaluate the effect of the increased number of nodes of the grapevine Cabernet Franc in relation to the interception of the photosynthetically active composition and its effect on the node fertility in high altitude region of Santa Catarina State. This experiment was conducted during the 2016/2017 harvest in a commercial vineyard located in the city of São Joaquim. Cabernet Franc plants grafted on the 'Paulsen 1103' rootstock were used. The treatments consisted of four different levels of retained node numbers: 15, 30,50 , and 75 nodes per plant. The experimental design was a randomized block design with four blocks and five plants per plot. The data were submitted to analysis of variance (ANOVA) and compared by the Tukey test with a $5 \%$ probability of error. The results of this study evidenced the importance of the interception of the photosynthetically active radiation in the nodes fertility, observing the reduction of the nodes fertility in situations of reduction of the interception of the photosynthetically active radiation, in this case, caused by the increase of the number of nodes, which resulted in a higher shoot number and branches, resulting in a denser canopy, and consequently reducing the interception of the photosynthetically active radiation that reaches the grape nodes, being this effect more pronounced in the buds located at the basal position of the branch.

KEYWORDS: Vitis vinifera L., pruning, vegetative balance, canopy management. 


\section{INTRODUÇÃO}

As regiões de elevada altitude de Santa Catarina (SC) caracterizam-se por apresentar vinhedos entre 900 e 1400 metros acima do nível do mar (WURZ et al. 2017a). Estas características favorecem o cultivo de variedade de uvas Vitis vinifera L., as quais atingem índices de maturação que permitem fornecer matéria prima para elaboração de vinhos diferenciados por sua intensa coloração, aroma e acidez (MARCON FILHO et al. 2015, MALINOVSKI et al. 2016). No entanto, observa-se nessa região baixo índice de fertilidade das gemas, em muitas ocasiões inferior à um cacho por gema (WURZ et al. 2017b), que associado a elevada disponibilidade hídrica (BEM et al. 2016) e solos com altos teores de matéria orgânica (MAFRA et al. 2011), promovem o excessivo crescimento vegetativo em detrimento do desempenho produtivo das videiras, sendo em muitos casos inferior a cinco toneladas/hectare (BRIGHENTI et al. 2014, WURZ et al. 2017b, WURZ et al. 2018).

Uma das alternativas viáveis para o aumento da produtividade está relacionada com o aumento da carga de gemas na poda invernal. A poda invernal é uma prática realizada anualmente em regiões de clima temperado, com o principal objetivo de regular a produção temporal da videira (MIELE \& MANDELLI 2012), sendo um manejo imprescindível e nele, ao aumentar a carga de gemas planta ${ }^{-1}$, é possível aumentar a produtividade do vinhedo e melhorar o equilíbrio vegeto-produtivo da videira.

O número de gemas que permanece nos sarmentos ou nos esporões após a poda determinará a dimensão da área foliar e o número de cachos de uvas. Vários estudos relataram diferenças, em função da intensidade e tipo de poda, no vigor vegetativo e composição final das bagas de uvas tanto para consumo in natura (CHRISTENSEN et al. 1994, AHMAD et al. 2004) quanto para vinificação (KURTURAL et al. 2006, BINDON et al. 2008, O'DANIEL et al. 2012).

Pesquisas pioneiras realizadas na Nova Zelândia por JACKSON \& LOMBARD (1993) descreveram que o número de gemas variando de 40 a 150 por plantas, em cinco cultivares, incluindo a Sauvignon Blanc, apresenta um comportamento curvilíneo, onde o aumento de 43 para 86 gemas por planta dobrou a produção, no entanto, ao aumentar o número de gemas para 150 por planta, o aumento da produção foi de apenas $12 \%$ em relação a 86 gemas por planta. Conforme trabalho realizado por GREVEN et al. (2015), o aumento de 24 para 72 gemas por planta resultou em aumento da produtividade de 4,8 para 12,7 ton/ha. No entanto, deve ser considerado ainda que o manejo da copa em função do tipo e intensidade da poda exerce efeito sobre a produção no ciclo seguinte, devido ao seu impacto sobre o conteúdo de reserva acumulado e à fertilidade das gemas (VASCONCELOS et al. 2009, PELLEGRINO et al. 2014).

Devido a uma carência de informações técnico-cientificas sobre o efeito da carga de gemas em relação a interceptação da radiação solar das gemas e o seu efeito na fertilidade de gemas, tem-se como objetivo desse trabalho avaliar o efeito do aumento da carga de gemas da videira 'Cabernet Franc' em relação a interceptação da redação fotossinteticamente ativa e o seu efeito na fertilidade de gemas em região de elevada altitude de Santa Catarina.

\section{MATERIAL E MÉTODOS}

Este experimento foi conduzido durante a safra 2016/2017, em um vinhedo comercial, localizado no

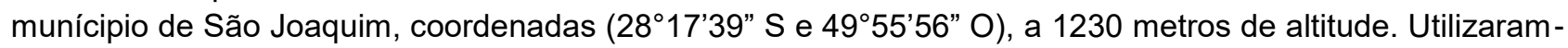
se plantas de 'Cabernet Franc' enxertadas sobre o porta-enxerto 'Paulsen 1103'. Os vinhedos foram implantados em 2004. O vinhedo caracteriza-se por apresentar plantas espaçadas de 3,0 x 1,5 m, em filas dispostas no sentido N-S, conduzidas em espaldeira, podadas em cordão esporonado duplo, a 1,2 m de altura e cobertas com tela de proteção anti-granizo, e com histórico de baixas produtividades.

Os solos da região enquadram-se nas classes Cambissolo Húmico, Neossolo Litólico e Nitossolo Háplico, desenvolvidos a partir de rocha riodacito e basalto (POTTER et al. 2004). O clima da região é classificado como 'Frio, Noites Frias e Úmido', Índice Heliotérmico de 1.714, precipitação pluvial média anual de $1.621 \mathrm{~mm}$ e a umidade relativa do ar média anual de 80\% (TONIETTO \& CARBONNAU 2004).

Os tratamentos consistiram em quatro diferentes níveis de cargas de gemas: 15, 30, 50 e 75 gemas planta $^{-1}$. A poda foi realizada para a 'Cabernet Franc' no dia 08 de setembro de 2016. Deixou-se 8, 15, 25 esporões com duas gemas cada esporão para os tratamentos 15, 30 e 50 gemas por planta, e para o tratamento 75 gemas por planta, deixou-se 30 esporões com duas gemas, e duas varas com oito gemas cada, sendo, portanto, este tratamento podado no sistema de poda mista, caracterizada pela presença de esporões e varas. Após a realização da poda, contabilizou-se o número de gemas por planta, cegando-se as que apresentavam valores acima de 15, 30, 50 e 75 gemas planta $^{-1}$.

Para a avaliação da fertilidade de gemas, foram coletados 20 ramos de um ano, de cada tratamento, no momento de dormência profunda das plantas, após a maturação das gemas, durante o inverno de 2017. 
O material vegetativo dormente foi levado imediatamente a Universidade do Estado de Santa Catarina, no Centro de Ciências Agroveterinárias - Lages, SC. As gemas foram individualizadas e separadas de acordo com suas posições no ramo em gemas basais $\left(1^{\underline{a}}\right.$ a $\left.3^{a}\right)$ e gemas medianas $\left(4^{\underline{a}}\right.$ a $\left.7^{\mathrm{a}}\right)$ e gemas apicais $\left(8^{\mathrm{a}}\right.$ a $\left.10^{a}\right)$. Cada segmento do ramo contendo uma gema foi disposto em bandejas de isopor com espuma fenólica hidratada.

As bandejas foram colocadas em câmara com temperatura, fotoperíodo e umidade controlados (Fitotron) com $60 \%$ de umidade relativa, temperatura de $20^{\circ} \mathrm{C}$ e 14 horas de luz por dia com intensidade de 300-400 $\mu \mathrm{E} \mathrm{m}^{-2} \mathrm{~s}^{-1}$ (ANDREINI et al. 2009). As gemas foram classificadas em férteis ou não férteis de acordo com a presença ou ausência da inflorescência.

Para avaliação da interceptação da radiação fotossinteticamente ativa, utilizou-se um ceptômetro AccuPAR (LP-80 Decagon, EUA). As medidas foram realizadas ao meio dia, quando o sol estava no zênite, durante os estádios fenológicos de plena florada, mudança de cor das bagas e maturidade, e os resultados expressos em PAR umol $\mathrm{m}^{-2} \mathrm{~s}^{-1}$. As leituras foram efetuadas em quatro posições diferentes: paralelo a linha das plantas; na zona dos cachos, perpendicular e paralelo a linha das plantas; no dossel vegetativo, perpendicular à linha das plantas.

Os dados meteorológicos foram obtidos a partir de Estação Meteorológica Automática Telemétrica do Centro de Informações de Recursos Ambientais e de Hidrometeorologia de Santa Catarina (EPAGRI/CIRAM), localizada na Estação Experimental da EPAGRI em São Joaquim

O delineamento experimental utilizado foi o de blocos ao acaso, com quatro blocos e cinco plantas por parcela. Os dados foram submetidos à análise de variância (ANOVA) e comparados pelo Teste Tukey a $5 \%$ de probabilidade de erro.

\section{RESULTADOS E DISCUSSÃO}

No período de maior crescimento vegetativo do vinhedo (novembro a março) no ciclo 2016/2017 a temperatura média foi de $17,3^{\circ} \mathrm{C}$, com uma precipitação acumulada de $532,7 \mathrm{~mm}$ (Figura 1). Entre os fatores que podem influenciar a fertilidade de gemas em videiras, a temperatura é a mais determinante. Normalmente, cultivares de espécies de videira americanas produzem inflorescências a temperaturas mais baixas (21 a $\left.22{ }^{\circ} \mathrm{C}\right)$ do que cultivares da espécie Vitis vinifera (27 a $\left.28{ }^{\circ} \mathrm{C}\right)$ (MULLINS et al. 2000). Nesse contexto, observou-se que os dados climáticos, em especial a temperatura média, ficou abaixo do ideal para diferenciação do primórdio floral na variedade vinífera, prejudicando a fertilidade das gemas.

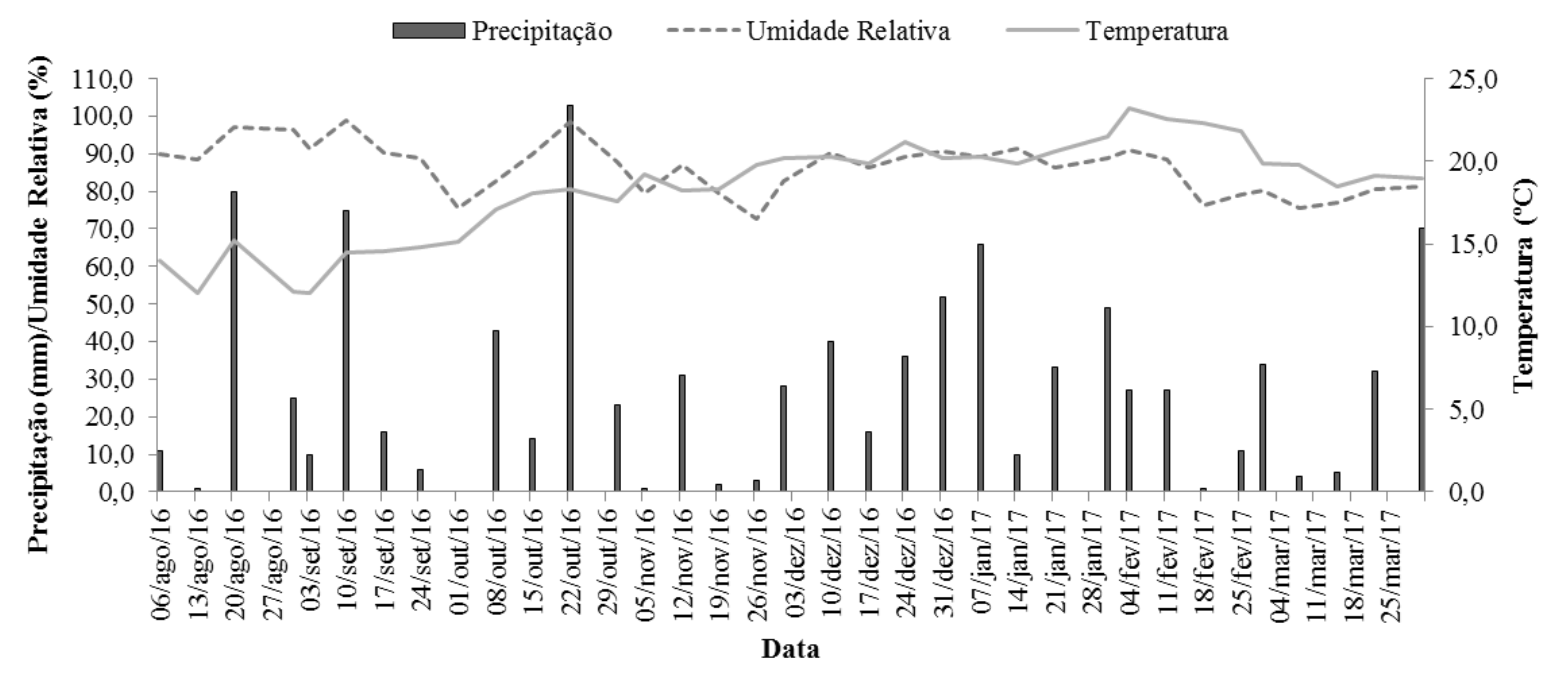

Figura 1. Precipitação pluviométrica acumulada $(\mathrm{mm})$, umidade relativa do ar $(\%)$ e temperatura média do ar $\left({ }^{\circ} \mathrm{C}\right)$ para São Joaquim, SC durante a safra 2016/2017. Fonte: EPAGRI/CIRAM.

Figure 1. Cumulative rainfall $(\mathrm{mm})$, relative air humidity (\%), and mean air temperature $\left({ }^{\circ} \mathrm{C}\right)$ for São Joaquim, SC during the 2016/2017 harvest.

Observou-se efeito do aumento da carga de gemas na interceptação da radiação fotossinteticamente ativa (Tabela 1). Em todas as posições avaliadas houve efeito da carga de gemas. De modo geral, ao aumentar a carga de gemas, observou-se uma redução da interceptação da radiação fotossinteticamente ativa na região onde localizam-se os cachos e no dossel vegetativo, sendo as maiores diferenças observadas entre as cargas de gemas, na posição do cacho (perpendicular à linha das plantas). 
Essa redução da interceptação da radiação solar interfere diretamente na fertilidade das gemas, como observado nas Tabelas 2 e 3.

Tabela 1. Efeito da carga de gemas na interceptação da radiação fotossinteticamente ativa no estádio fenológico plena florada na videira Cabernet Franc (Vitis vinífera L.) em região de elevada altitude de Santa Catarina. Safra 2017.

Table 1. Effect of retained node number on the interception of the photosynthetically active radiation in the full flowering phenological stage of the Cabernet Franc (Vitis vinifera L.) grapevine in a high-altitude region of Santa Catarina. 2017 Harvest.

\begin{tabular}{lcccc}
\hline Radiação Fotossintética Ativa (PAR) & \multicolumn{4}{c}{ Carga de Gemas } \\
\cline { 2 - 5 } & 15 & 30 & 50 & 75 \\
\hline Cacho (perpendicular à linha das plantas) & $821.49 \mathrm{a}$ & $89.22 \mathrm{~b}$ & $42.69 \mathrm{~b}$ & $63.38 \mathrm{~b}$ \\
Cacho (paralelo a linha das plantas) & $521.54 \mathrm{a}$ & $138.84 \mathrm{~b}$ & $117.40 \mathrm{~b}$ & $92.23 \mathrm{~b}$ \\
Dossel (perpendicular à linha das plantas) & $723.97 \mathrm{a}$ & $239.99 \mathrm{ab}$ & $178.58 \mathrm{~b}$ & $136.36 \mathrm{~b}$ \\
\hline
\end{tabular}

*Médias seguidas da mesma letra, na linha, não diferem entre si pelo teste Tukey a $5 \%$ de probabilidade de erro. ns = não significativo pela análise de variância (ANOVA) a 5\% de probabilidade de erro.

A formação da gema fértil é a consequência da diferenciação do primórdio indiferenciado em primórdio reprodutivo (BOTELHO et al. 2006), sendo a intensidade luminosa um dos fatores climáticos mais limitante para a formação de gemas férteis. Condições insatisfatórias de luz durante a iniciação da inflorescência reduz severamente a fertilidade de gemas e ramos mais expostos à luz, normalmente são mais férteis (KELLER \& KOBLET 1995). Diversos estudos citam a redução da radiação solar como principal fator responsável pela redução da fertilidade de gemas em plantas podadas com maior carga de gemas/planta, visto que dentre os fatores que influenciam a fertilidade de gemas, destaca-se a temperatura e radiação solar (VASCONCELOS et al. 2009, GREVEN et al. 2014). Trabalhos realizados por WURZ et al. (2017b) e WURZ et al. (2018), observaram que ao desfolhar a videira na região dos cachos, expondo as gemas a uma maior radiação solar, resulta em aumento da fertilidade de gemas da videira.

As diferentes cargas de gemas planta ${ }^{-1}$ nas plantas de Cabernet Franc influenciaram a fertilidade de gemas, sendo esta avaliada de duas formas: (a) \% de gemas férteis (Tabela 2) e (b) cachos gemas ${ }^{-1}$ (Tabela 3).

Tabela 2. Efeito da carga de gemas na fertilidade de gemas (\% gemas férteis) da videira Cabernet Franc (Vitis vinífera L.) em região de elevada altitude de Santa Catarina. Safra 2017.

Table 2. Effect of retained node number on node fertility (\% fertile buds) of Cabernet Franc (Vitis vinifera L.) grapevine in high altitude region of Santa Catarina. 2017 Harvest.

\begin{tabular}{|c|c|c|c|c|c|}
\hline \multirow{2}{*}{$\begin{array}{c}\text { Fertilidade } \\
\text { (\% gemas férteis) }\end{array}$} & \multicolumn{4}{|c|}{ Carga de Gemas } & \multirow{2}{*}{$\begin{array}{l}\text { CV } \\
(\%)\end{array}$} \\
\hline & 15 & 30 & 50 & 75 & \\
\hline Gemas Basais & $94,3 \mathrm{a}$ & $94,3 \mathrm{a}$ & $88,6 a b$ & $66,3 \mathrm{~b}$ & 13,4 \\
\hline Gemas Medianas & $100,0 \mathrm{a}$ & $100,0 \mathrm{a}$ & $93,6 \mathrm{~b}$ & $80,9 \mathrm{c}$ & 2,9 \\
\hline Gemas Apicais & $97,2 \mathrm{a}$ & $89,7 \mathrm{~b}$ & $97,2 \mathrm{a}$ & $91,5 \mathrm{ab}$ & 5,6 \\
\hline
\end{tabular}

${ }^{*}$ Médias seguidas da mesma letra, na linha, não diferem entre si pelo teste Tukey a $5 \%$ de probabilidade de erro. ns = não significativo pela análise de variância (ANOVA) a 5\% de probabilidade de erro.

Ao comparar a posição distal das gemas, observou-se uma tendência da maior fertilidade nas gemas medianas e apicais. Para WINKLER (1965), há maior acúmulo de carboidratos nessas regiões medianas e apicais do ramo, e nesse contexto, tem-se uma tendência do aumento da fertilidade nessa porção do ramo. Trabalhos realizados por MUNHOZ et al. (2016), com as variedades Greco di Tufo e Coda di Volpe e por ROSA et al. (2014), com as variedades Cabernet Sauvignon e Nebbiolo cultivadas em regiões de elevada altitude de Santa Cantarina, observaram baixa fertilidade de gemas na região basal dos ramos das variedades estudadas.

Quando comparou-se as diferentes cargas de gemas, observou-se uma redução da fertilidade de gemas (\% gemas férteis) nas cargas de 50 e 75 gemas/planta nas gemas basais e medianas. Na avaliação das gemas apicais, observou-se apenas para a carga de 30 gemas uma redução da fertilidade de gemas (\% gemas férteis), não diferindo estatisticamente da carga de 75 gemas/planta. Essa redução da fertilidade de gemas, está associado ao maior adensamento do dossel vegetativo, reduzindo a interceptação da radiação 
fotossinteticamente ativa, e consequentemente reduzindo a fertilidade de gemas. Para BRIGHENTI et al. (2017), adaptando o tipo de poda com a posição das gemas mais férteis é possível aumentar a produtividade, pois haverá um aumento no número de gemas férteis ao adotar o sistema de poda adequado para cada variedade, e em cada manejo da videira.

Tabela 3. Efeito da carga de gemas na fertilidade de gemas (número de cachos gemas ${ }^{-1}$ ) da videira Cabernet Franc (Vitis vinífera L.) em região de elevada altitude de Santa Catarina. Safra 2017.

Table 3. Effect of retained node number on node fertility (number of nodes ${ }^{-1}$ bunches) of Cabernet Franc (Vitis vinifera L.) grapevine in the high-altitude region of Santa Catarina. 2017 Harvest.

\begin{tabular}{|c|c|c|c|c|c|}
\hline \multirow{2}{*}{$\begin{array}{l}\text { Fertilidade } \\
\left(\text { Cachos gemas }^{-1)}\right.\end{array}$} & \multicolumn{4}{|c|}{ Carga de Gemas } & \multirow{2}{*}{$\begin{array}{l}\text { CV } \\
(\%)\end{array}$} \\
\hline & 15 & 30 & 50 & 75 & \\
\hline Gemas Basais & $1,4 a$ & $1,3 a b$ & $1,2 a b$ & $0,8 \mathrm{~b}$ & 19,9 \\
\hline Gemas Medianas & $1,8 \mathrm{a}$ & $1,7 a b$ & $1,5 \mathrm{~b}$ & $1,1 \mathrm{c}$ & 9,4 \\
\hline Gemas Apicais & $1,8 \mathrm{~ns}$ & 1,6 & 1,5 & 1,4 & 13,5 \\
\hline
\end{tabular}

*Médias seguidas da mesma letra, na linha, não diferem entre si pelo teste Tukey a $5 \%$ de probabilidade de erro. ns = não significativo pela análise de variância (ANOVA) a 5\% de probabilidade de erro.

Observou-se efeito da carga de gemas/planta na fertilidade de gemas avaliada através do número de cachos gema-1 (Tabela 3). De modo geral, verificou-se uma redução do número de cachos gema $^{-1} \mathrm{com}^{\circ}$ aumento da carga de gemas/planta. Na avaliação das gemas basais, observou-se média de 1,4, 1,3 e 1,2 cachos gema- ${ }^{-1}$ para as cargas de 15,30 e 50 gemas/planta, enquanto para a carga de 75 gemas/planta observou-se média de 0,8 cachos gema-1 ${ }^{-1}$ Comportamento similar foi observado nas gemas medianas, observou-se média de 1,8, 1,7 1,5 cachos gema $^{-1}$, enquanto para a carga de 75 gemas/planta apresentou 1,1 cachos gema-1 ${ }^{-1}$ Para as gemas localizadas na posição distal do ramo, não se observou efeito da carga de gemas na fertilidade de gemas (cacho gema $^{-1}$ ), variando de 1,8 a 1,4 cachos gema-1, para as cargas de 15 e 75 gemas/planta, respectivamente.

Assim como na variável fertilidade de gemas, avaliada através da \% de gemas férteis, para a variável número de cachos gema-1, observou-se efeito significativo do aumento da carga de gemas para a fertilidade de gemas, avaliada por meio do número de cachos gema ${ }^{-1}$.

Os resultados desse estudo evidenciam a importância da interceptação da radiação fotossinteticamente ativa na fertilidade de gemas, observando redução da fertilidade de gemas em situações de redução da interceptação da radiação fotossinteticamente ativa, nesse caso, causado pelo aumento da carga de gemas, que resultado em um maior número de brotação e ramos, ocasionado um dossel mais denso, e consequentemente reduzindo a interceptação da radiação fotossinteticamente ativa que alcança as gemas da videira, sendo esse efeito, mais pronunciado nas gemas localizada na posição basal do ramo.

Portanto, ao adotar sistemas de poda com carga de gemas alta ( $>50$ gemas planta-1 $)$, torna-se imprescindível a adoção de técnicas de manejo com objetivo de favorecer uma melhor interceptação da radiação fotossinteticamente ativa no dossel vegetativo, como por exemplo, a desfolha da videira.

\section{CONCLUSÃO}

O aumento da carga de gemas resulta em redução da radiação fotossinteticamente ativa no dossel vegetativo da videira Cabernet Franc.

A redução da radiação fotossinteticamente ativada no dossel vegetativo influencia a fertilidade de gemas.

Ocorre redução da fertilidade das gemas basais ao aumentar o número da carga de gemas planta-1.

\section{REFERÊNCIAS}

AHMAD MW et al. 2004. Effect of pruning severity on growth behavior of spur and bunch morphology of grapes (Vitis vinifera L.) cv. Perlette. International Journal of Agriculture and Biology 6: 160-161.

ANDREINI L et al. 2009. Study on the morphological evolution of bud break in Vitis vinifera L. Vitis 48: 153-158.

BEM BP et al. 2016. Effect of four training systems on the temporal dynamics of downy mildew in two grapevine cultivars in southern Brazil. Tropical Plant Pathology 41: 370-379.

BINDON K et al. 2008. Influence of partial root zone drying on the composition and accumulation of anthocyanins in grape berries (Vitis vinifera cv. Cabernet sauvignon). Australian Journal and Grape Wine Research 14: 91-103.

BOTELHO RV et al. 2006. Fertilidade de Gemas de Videira: Fisiologia e fatores envolvidos. Ambiência 2: $129-144$.

BRIGHENTI AF et al. 2014. Desempenho vitícola de variedades autóctones italianas em condição de elevada altitude no 
Sul do Brasil. Pesquisa Agropecuária Brasileira 49: 465-474.

BRIGHENTI AF et al. 2017. Ecophysiology of three Italian cultivars subjected to two pruning methods in Santa Catarina, Brazil. Acta Horticulturae 1: 381-388.

CHRISTENSEN LP et al. 1994. The effects of pruning level and post bud break cane adjustment on Thompson seedless raisin production and quality. American Journal of Enology and Viticulture 45: 141-152.

GREVEN MM et al. 2014. Influence of retained node number on Sauvignon Blanc grapevine vegetative growth and yield. Australian Journal of Grape and Wine Research 20: 263-271.

GREVEN MM et al. 2015. Influence of retained node number on Sauvignon Blanc grapevine phenology in a cool climate. Australian Journal of Grape and Wine Research 21: 209-301.

JACKSON DI \& LOMBARD PB. 1993. Environmental and management practices affecting grape composition and wine quality - a review. American Journal of Enology and Viticulture 44: 409-430.

KELLER M \& KOBLET W. 1995. Dry matter and leaf area partitioning, bud fertility and second season growth of Vitis vinifera L.: Responses to nitrogen supply and limiting irradiance. Vitis 34: 77-83.

KURTURAL SK et al. 2006. Effects of pruning and cluster thinning on yield and fruit composition of 'Chambourcin' grapevines. HortTechnology 16: 233-240.

MAFRA SHM et al. 2011. Atributos químicos do solo e estado nutricional de videira Cabernet Sauvignon (Vitis vinifera L.) na Serra Catarinense. Revista de Ciências Agroveterinárias 10: 44-53.

MALINOVSKI LI et al. 2016. Viticultural performance of Italian grapevines in high altitude regions of Santa Catarina State, Brazil. Acta Horticulturae 1115: 203-210.

MIELE A \& MANDELLI F. 2012. Manejo do dossel vegetativo e seu efeito nos componentes de produção da videira Merlot. Revista Brasileira de Fruticultura 34: 964-973.

MARCON FILHO JL et al. 2015. Raleio de cachos sobre o potencial enológico da uva 'Cabernet Franc' em duas safras. Ciência Rural 45: 2150-2156.

MULLINS MG et al. 2000. Biology of the grapevine. Cambridge: University Press. 239p.

MUNHOZ B et al. 2016. Fertilidade e Análise de gemas das videiras Greco di Tufo, Coda di Volpe e Viognier cultivadas em São Joaquim - Santa Catarina. Revista Agropecuária Catarinense 29: 68-72.

O'DANIEL SB et al. 2012. Effects of balanced pruning severity on Traminette (Vitis spp.) in a warm climate. American Journal of Enology and Viticulture 63: 284-290.

PELLEGRINO A et al. 2014. Management practices impact vine carbohydrate status to a greater extent than vine productivity. Frontiers in Plant Science 5: 1-13.

ROSA AM et al. 2014. Fertilidade e reserva de carbono e nitrogênio em gemas de ramos das viníferas 'Cabernet Sauvignon' e 'Nebbiolo'. Revista Brasileira de Fruticultura 36: 576-585.

POTTER RO et al. 2004. SOLOS do Estado de Santa Catarina. Rio de Janeiro: Embrapa Solos, 726p. (Embrapa Solos. Boletim de pesquisa e desenvolvimento, 46).

TONIETTO J \& CARBONNEAU A. 2004. A multicriteria climatic classification system for grape-growing regions worlwide. Agricultural and Forest Meteorology 124: 81-97.

VASCONCELOS MC et al. 2009. The flowering process of Vitis vinifera: a review. American Journal of Enology and Viticulture 60: 411-434.

WINKLER AJ. 1965. Viticultura. México: Continental. 792p.

WURZ DA et al. 2017a. New wine-growing regions of Brazil and their importance in the evolution of Brazilian wine. BIO Web of Conferences 9: 1-4.

WURZ DA et al. 2017b. Agronomic performance of 'Cabernet Sauvignon' with leaf removal management in a highaltitude region of Southern Brazil. Pesquisa Agropecuária Brasileira 52: 869-876.

WURZ DA et al. 2018. Época de desfolha e sua influência no desempenho vitícola da uva 'Sauvignon Blanc' em região de elevada altitude. Revista de Ciências Agroveterinárias 17: 91-99. 